\title{
Downregulation of SYT7 inhibits glioblastoma growth by promoting cellular apoptosis
}

\author{
BING XIAO $^{1 *}$, JIANBIN LI $^{2 *}$, YANGHUA FAN $^{1}$, MINHUA YE $^{1}$, SHIGANG LV $^{1}$, \\ $\mathrm{BIN} \mathrm{XU}^{1}$, YI CHAI ${ }^{1}$, ZHIQING ZHOU ${ }^{3}$, MIAOJING WU ${ }^{1}$ and XINGEN ZHU ${ }^{1}$ \\ ${ }^{1}$ Department of Neurosurgery, The Second Affiliated Hospital of Nanchang University, Nanchang, Jiangxi 330006; \\ ${ }^{2}$ Department of Neurosurgery, The Second Hospital of Nanchang, Nanchang, Jiangxi 330003; ${ }^{3}$ Department of \\ Oncology, The Second People's Hospital of Huaihua City, Huaihua, Hunan 418000, P.R. China
}

Received December 20, 2016; Accepted August 17, 2017

DOI: $10.3892 / \mathrm{mmr} .2017 .7723$

\begin{abstract}
Synaptotagmin-7 (SYT7) is a member of the synaptotagmin gene family, and encodes a protein that mediates the calcium-dependent regulation of membrane trafficking during synaptic transmission. A previous study demonstrated that the expression of SYT7 is associated with prostate cancer and serves an important role in development of prostate cancer. However, the roles of SYT7 in the progression of glioma remain unknown. In the present study, reverse transcription-quantitative polymerase chain reaction (RT-qPCR) analysis demonstrated that SYT7 was expressed in three human glioma cell lines. Western blotting and RT-qPCR analysis demonstrated the knockdown efficiency of SYT7 shRNA in 293T cells and U87MG cells. Celigo Image Cytometer Analysis, a caspase-3/7 assay, flow cytometry and an MTT assay demonstrated that the proliferation of U87MG cells was inhibited as SYT7 was downregulated by a lentiviral vector expressing SYT7 shRNA, via the promotion of cellular apoptosis. The results of the present study demonstrated that the downregulation of SYT7 inhibited glioblastoma growth by promoting cellular apoptosis, and that SYT7 may therefore be a potential target for glioma intervention.
\end{abstract}

\section{Introduction}

Glioblastoma is a common type of malignant tumor in the central nervous system, characterized by aggressive behavior, rapid progression, high recurrence rates and poor prognosis.

Correspondence to: Dr Miaojing Wu or Professor Xingen Zhu, Department of Neurosurgery, The Second Affiliated Hospital of Nanchang University, 1 Minde Road, Nanchang, Jiangxi 330006, P.R. China

E-mail: 905327378@qq.com

E-mail: zxg2008vip@163.com

*Contributed equally

Key words: synaptotagmin-7, glioblastoma, proliferation, apoptosis
The median survival of patients with glioblastoma patients is $<1$ year (1-4). Although progress has been made in the biological research into and treatment of glioblastoma, the primary treatment for glioblastoma is surgery combined with postoperative radiotherapy, chemotherapy and immunotherapy; however, the overall therapeutic effect remains poor. Knowledge about this malignant tumor is limited, and its exact pathogenesis remains unclear. Therefore, it is of importance to examine the molecular mechanism underlying the progression of glioblastoma.

The mammalian synaptotagmin (SYT) family consists of 17 isoforms. SYT proteins are sensors of calcium signaling in cellular secretions (5-8). SYT consists of a transmembrane helix close to the $\mathrm{N}$-terminus, a flexible region of variable length, and two C2 domains (9), with an average length of $\sim 135$ amino acid residues, and each of them is composed of eight $\beta$-folding and three ring structures. SYT1-SYT7 are all expressed in the brain, particularly SYT1, SYT2, SYT3 and SYT7 (10). SYT7 is widely distributed in different types of biological tissue, and it may be spliced to $>12$ SYT7 variants with differing genetic structures. SYT7 is located in core vesicles, opposite the reticular structures of the Golgi apparatus and lysosomes in PC12 cells (11). SYT is able to interact with soluble $N$-ethylmaleimide-sensitive fusion protein attachment protein receptor (SNARE) to regulate fusion of the vesicle membrane and plasma membrane, which serves an important role in cell secretion (12). A previous study demonstrated that SYT7 is able to regulate apoptosis, cell differentiation, cytokine activity, amine biosynthesis, transcriptional regulation and vesicular transport (13). SYT7 is involved in lysosome-mediated repair mechanisms and induces apoptosis (14), and it is associated with prostate cancer and serves an important role in prostate cancer development (15). However, the roles of SYT7 in the progression of glioma remain unknown.

The present study demonstrated that SYT7 was expressed in glioblastoma, and that the proliferation of U87MG cells was inhibited as SYT7 was downregulated by a lentiviral vector expressing SYT7 short hairpin (sh)RNA, via the promotion of cellular apoptosis. The results of the present study demonstrated that downregulation of SYT7 may inhibit glioblastoma growth by promoting cellular apoptosis. These results enhance 
the possibility that SYT7 may be a therapeutic target for glioblastoma treatment.

\section{Materials and methods}

Cell culture and reagents. The human glioma cell lines A172 (cat. no. TCHu171), U251 (cat. no. TCHu58) and U87 (cat. no. TCHu138), and human epithelial 293T cells (cat. no. GNHu17) (all from Cell Bank of the Chinese Academy of Sciences, Shanghai, China) were cultured in Dulbecco's modified Eagle's medium (DMEM; Hyclone; GE Healthcare Life Sciences, Logan, UT, USA) supplemented with $10 \%$ heat-inactivated fetal bovine serum (Hyclone; GE Healthcare Life Sciences) at $37^{\circ} \mathrm{C}$ in an atmosphere which contains $5 \% \mathrm{CO}_{2}$. The U87 cell line employed in the present study is known to be misidentified/cross-contaminated with an unknown cell line, which is most likely also a glioblastoma cell line (16).

Reverse transcription-quantitative polymerase chain reaction (RT-qPCR) assay. Total RNA was extracted from the three glioma cell lines (A172, U251 and U87) using the TRIzol total RNA reagent (Invitrogen; Thermo Fisher Scientific, Inc., Waltham, MA, USA). cDNA synthesis was performed using $2 \mu \mathrm{g}$ total RNA using the RevertAid ${ }^{\mathrm{TM}} \mathrm{H}$ Minus First Strand cDNA Synthesis kit (Takara Bio, Inc., Otsu, Japan). The SYT7 primers were obtained from Shanghai GeneChem Co., Ltd. (Shanghai, China), and GAPDH was used as an internal control. The primers were as follows: SYT7 forward, 5'-ACT CCATCATCGTGAACATCATC-3' and reverse, 5'-TCGA AGGCGAAGGACTCATTG-3'; GAPDH forward, 5'-TGA CTTCAACAGCGACACCCA-3' and reverse, 5'-CACCCT GTTGCTGTAGCCAAA-3'. qPCR was performed using the SYBR PrimeScript qPCR kit (Takara Bio, Inc.) on an Applied Biosystems 7300 Fluorescent Quantitative PCR System (Applied Biosystems; Thermo Fisher Scientific, Inc.). The reaction mixtures were incubated at $95^{\circ} \mathrm{C}$ for $30 \mathrm{sec}$, with 45 amplification cycles at $95^{\circ} \mathrm{C}$ for $5 \mathrm{sec}, 60^{\circ} \mathrm{C}$ for $30 \mathrm{sec}$ and $72^{\circ} \mathrm{C}$ for $60 \mathrm{sec}$, followed by a final extension of $72^{\circ} \mathrm{C}$ for $7 \mathrm{~min}$. The PCR products of SYT7 and GAPDH were 178 and $121 \mathrm{bp}$, respectively. The relative expression of SYT7 mRNA was calculated via the $2^{-\Delta \Delta C q}$ method (17), using the GAPDH mRNA expression level for normalization.

Construction of the shRNA lentiviral vector and transfection. The lentiviruses expressing shRNA targeting the sequence of the SYT7 gene (5'-TCACCGTGAAGATCATGAA-3') and negative control shRNA were purchased from Shanghai GeneChem Co., Ltd. The shRNAs were subsequently cloned into a pGCSIL-green fluorescent protein lentiviral vector with $A g e \mathrm{I} / E c o$ RI sites to generate recombinant the lentiviral shRNA expression vectors. Lentiviral vectors and packaging vectors were transfected into $293 \mathrm{~T}$ cells using Lipofectamine 2000, according to manufacturer's instructions. Lentiviral particles were purified using ultracentrifugation, and an endpoint dilution assay was performed to determine the titer of the lentiviruses. The U87MG glioblastoma cells, seeded in 6-well plates at a density of $4.0 \times 10^{5}$ cells/well, were infected with shSYT7-lentivirus $\left(4 \times 10^{8} \mathrm{TU} / \mathrm{ml} \times 2.5 \mu \mathrm{l}\right)$ or negative control $\left(8 \times 10^{8} \mathrm{TU} / \mathrm{ml} \times 1.25 \mu \mathrm{l}\right)$ lentivirus. The cells were observed under a fluorescence microscope (MicroPublisher 3.3RTV; Olympus, Tokyo, Japan) 72 h subsequently. Following 5 days of infection, the knockdown efficiency of the shRNA-SYT7 vectors was investigated via qPCR analysis.

Cell growth assay. Cell growth was measured using a Celigo Image Cytometer (Nexcelom Bioscience LLC, Lawrence, MA, USA). U87MG cells at the logarithmic phase following infection with the shCtrl lentivirus or shSYT7 lentivirus were seeded at $2 \times 10^{3}$ cells/well in 96-well plates, 3 duplicates/group, and incubated at $37^{\circ} \mathrm{C}$ for 5 days. The cells in the plates were counted using the Celigo Image Cytometer for each day's analysis.

Caspase-3/7 assay. U87MG cells at the logarithmic phase following infection with the shCtrl lentivirus or shSYT7 lentivirus were seeded in a 96-well plate and incubated at $37^{\circ} \mathrm{C}$ for 5 days in a $5 \% \mathrm{CO}_{2}$ incubator. Caspase-Glo3/7 (Promega, Madison, WI, USA) buffer and powder were mixed to generate the Caspase-Glo reaction buffer and, following cell counting, the cell suspension was adjusted to a concentration of $1 \times 10^{4}$ cells/well at room temperature. Cells were seeded in a new 96-well plate and a control group was designated; control cells were plated in the same manner however, they were not treated with reaction buffer. A total of $100 \mu 1$ Caspase-Glo reaction buffer was added to each well, the cells were incubated for $2 \mathrm{~h}$ at $37^{\circ} \mathrm{C}$, and the signal intensity was measured using a Multiskan Spectrum microplate reader at $480 \mathrm{~nm}$ (Tecan Group Ltd., Männedorf, Switzerland). All experiments were performed in triplicate.

Apoptosis assay using flow cytometry. Annexin V-allophycocyanin (APC) single labeling was performed to detect cellular apoptosis. U87MG cells were infected with shSYT7 or shCtrl lentiviruses and incubated at $37^{\circ} \mathrm{C}$. U87MG cells were harvested by trypsinization and rinsed in $1 \mathrm{X}$ binding buffer, followed by the addition of $10 \mu \mathrm{l}$ Annexin V-APC buffer (eBioscience; Thermo Fisher Scientific, Inc.) for $10 \mathrm{~min}$ staining at room temperature. A FACSCalibur II cell sorter and CellQuest Research Software (version 5.1; BD Biosciences, San Diego, CA, USA) were used for the assay. All experiments were performed in triplicate.

MTT cell proliferation assay. An MTT Cell Proliferation Assay kit (American Type Culture Collection, Manassas, VA, USA) was used for the cell proliferation assay, according to the manufacturer's instructions. Cells were rinsed with PBS at the end of the culture period and the MTT reagents were added to the 96-well plate according to the manufacturer's instructions. DMSO was then used to dissolve the formazan and the absorbance was measured using a microplate reader at $570 \mathrm{~nm}$. All experiments were repeated three times.

Western blot analysis. The cells were harvested at $48 \mathrm{~h}$ following transfection and lysed in radioimmunoprecipitation assay buffer (Beyotime Institute of Biotechnology, Shanghai, China) for $30 \mathrm{~min}$ at $4^{\circ} \mathrm{C}$. Proteins were extracted and the concentration was determined using a Bicinchoninic Acid Protein Assay kit (Pierce; Thermo Fisher Scientific, 
Inc.). Equal amounts of total protein $(40 \mu \mathrm{g})$ of each group was separated by SDS-PAGE on a $12.5 \%$ gel according to the Laemmli method, as described by Liu et al (18), and transferred to polyvinylidene fluoride membranes. Membranes were blocked in TBST buffer (containing 0.1\% Tween-20) with $5 \%$ skim milk at room temperature for $1 \mathrm{~h}$, and then incubated with the following primary antibodies overnight at $4^{\circ} \mathrm{C}$ : Polyclonal mouse anti-SYT7 (cat. no. SAB1412773, 1:2,000; Sigma-Aldrich; Merck KGaA, Darmstadt, Germany) and anti-GAPDH antibody (cat. no. sc-47724, 1:2,000; Santa Cruz Biotechnology, Inc., Dallas, TX, USA). Goat anti-mouse immunoglobulin G conjugated with horseradish peroxidase (cat. no. sc-2005; Santa Cruz Biotechnology, Inc.) was used as the secondary antibody at a 1:2,000 dilution for a $2 \mathrm{~h}$ incubation at room temperature. The bound antibodies were detected using an Enhanced Chemiluminescence Plus Western Blotting Detection System (GE Healthcare Life Sciences, Little Chalfont, UK). GAPDH was used as an internal control to normalize the SYT7 expression levels.

Statistical analysis. Data were statistically analyzed using a $\chi^{2}$ test (for PCR, caspase-3/7 assay and FACS analysis) and one-way analysis of variance with Scheffe's post hoc test, using the SPSS 20.0 statistical software package (IBM Corp., Armonk, NY, USA). Data are presented as the mean \pm standard deviation. $\mathrm{P}<0.05$ was considered to indicate a statistically significant difference.

\section{Results}

Expression of SYT7 in three human glioma cell lines. The expression levels of SYT7 were examined in three glioma cell lines (A172, U251 and U87). The expression levels of SYT7 mRNA were evaluated by RT-qPCR analysis. The results demonstrated that SYT7 was expressed in all three human glioma cell lines (Fig. 1). GAPDH was used as an internal control.

Knockdown efficiency in 293 Tells as determined by western blotting. In order to determine the target knockdown efficiency and exogenous selection, 293T cells were infected with shSYT7 lentivirus and shCtrl lentivirus, and the SYT7 protein expression levels were measured by western blotting. It was observed that the protein expression level of SYT7 was decreased by shSYT7 compared with the shCtrl group (Fig. 2).

shSYT7 works efficiently in U87MG cells. U87MG cells were infected with shSYT7 lentivirus and shCtrl lentivirus in order to demonstrate the efficacy of shSYT7 in U87MG cells. The mRNA expression level of SYT7 was significantly downregulated by shSYT7; treatment with shRNA decreased the level of SYT7 mRNA to $20 \%$ of the control value (Fig. 3), as determined by qPCR analysis.

Cell proliferation is inhibited by shSYT7 by promoting cellular apoptosis. To examine the effect of SYT7 on cell proliferation, U87MG cells were infected with shSYT7 and shCtrl lentiviruses. Cells were seeded at $2 \times 10^{3}$ cells/well in

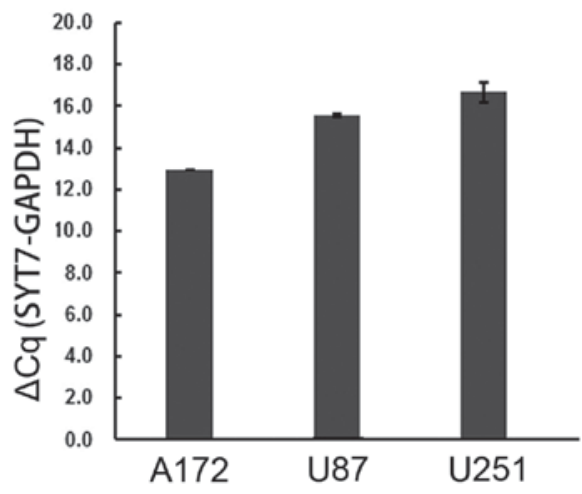

Figure 1. Expression of SYT7 in three human glioma cell lines. SYT7 was expressed in all three human glioma cell lines. GAPDH was used as an internal control. SYT7, synaptotagmin-7.

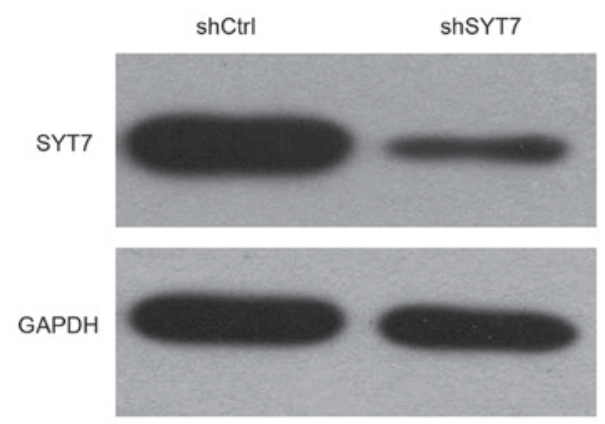

Figure 2. Knockdown efficiency in $293 \mathrm{~T}$ cells, assayed by western blotting. The protein expression level of SYT7 was decreased by shSYT7 compared with the shCtrl group. sh, short hairpin; SYT7, synaptotagmin-7.

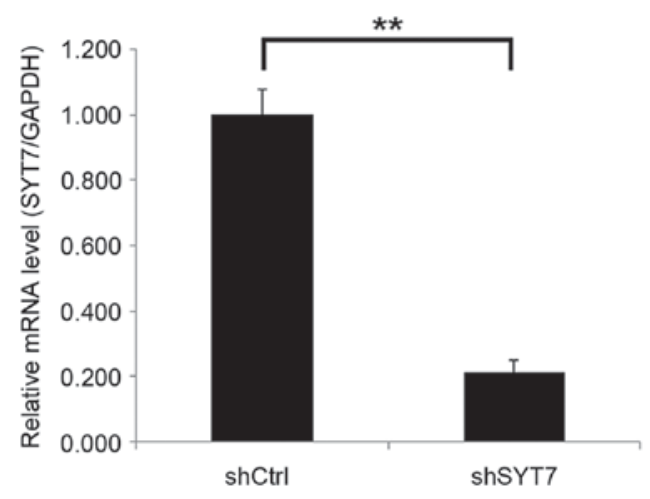

Figure 3. shSYT7 works efficiently in U87MG cells. A total of 3 days following shSYT lentivirus infection, the mRNA expression level of SYT7 was significantly downregulated by shSYT7 compared with the shCtrl group. ${ }^{* *} \mathrm{P}<0.01$. sh, short hairpin; SYT7, synaptotagmin-7; Ctrl, control.

96-well plates and counted using the Celigo Image Cytometer for 5 days. It was observed that U87MG cell growth was inhibited by shSYT7 compared with the shCtrl group (Fig. 4). In addition, an MTT cell proliferation assay was performed 3 days subsequent to infection. It was observed that the results were consistent with the Celigo Image Cytometer Analysis. U87MG cell proliferation was markedly inhibited by shSYT7 compared with the shCtrl group, with a relative survival of $33.8 \%$ of the control (Fig. 5). To examine the mechanism underlying cell growth inhibition, a 

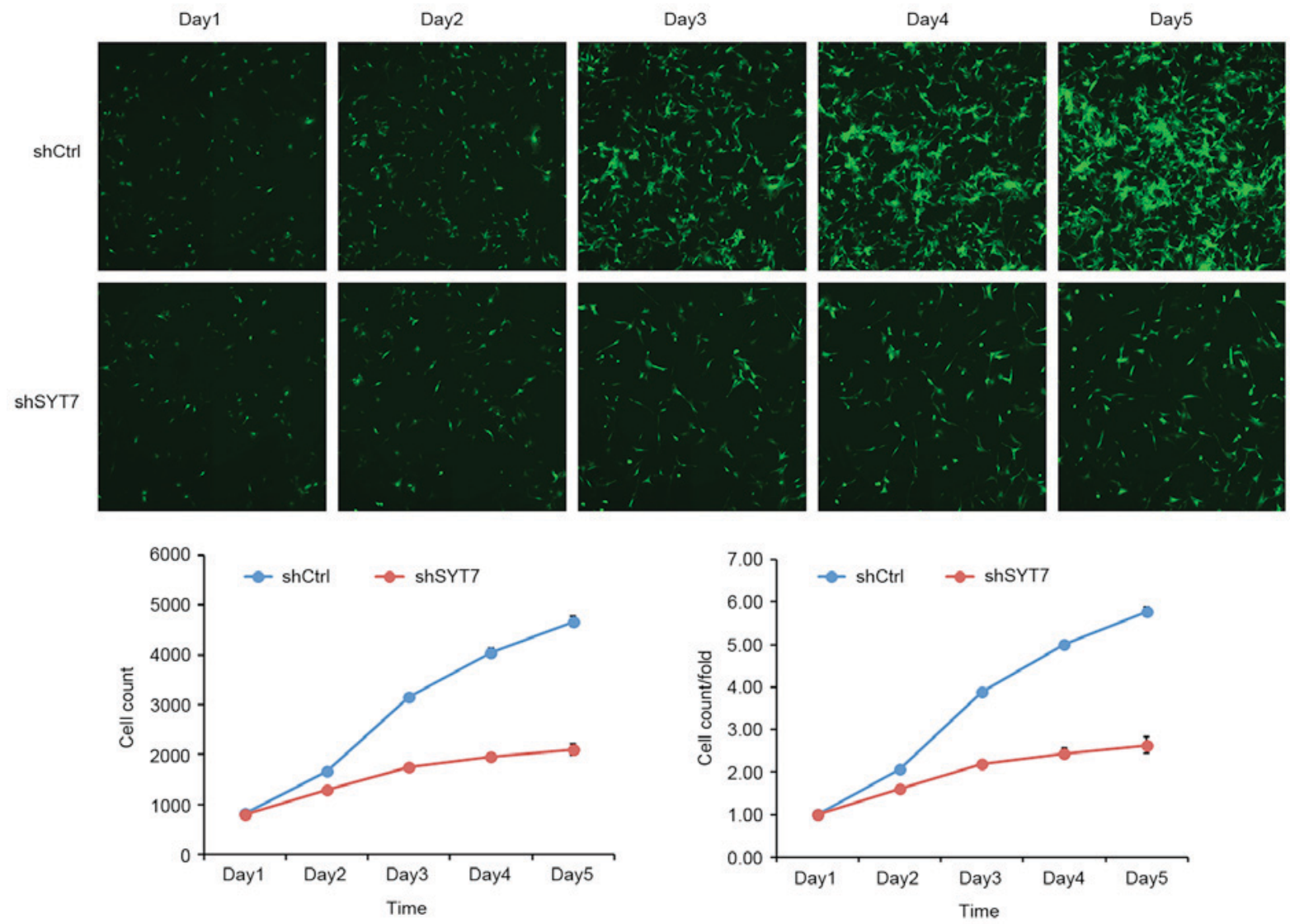

Figure 4. Effect of SYT7 on cell proliferation, as assayed by Celigo Image Cytometer analysis. U87MG cells were infected with the shSYT7 lentivirus or shCtrl lentivirus and seeded in 96-well plates. The cells were counted for 5 days. U87MG cell growth was inhibited by shSYT7 compared with the shCtrl group. Magnification, x100. sh, short hairpin; SYT7, synaptotagmin-7; Ctrl, control.
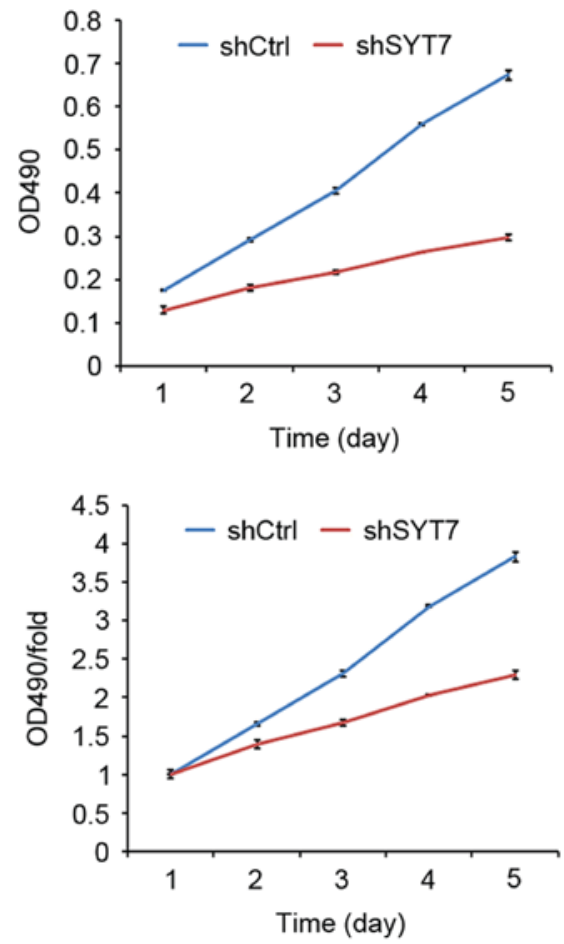

Figure 5. Effect of SYT7 on cell proliferation, as determined by MTT assay. U87MG cells were infected with the shSYT7 lentivirus or shCtrl lentivirus. A total of 3 days following infection, cells were seeded in 96-well plates and assayed each day for 5 days. U87MG cell growth was inhibited by shSYT7 compared with the shCtrl group. sh, short hairpin; SYT7, synaptotagmin-7; Ctrl, control; OD, optical density.

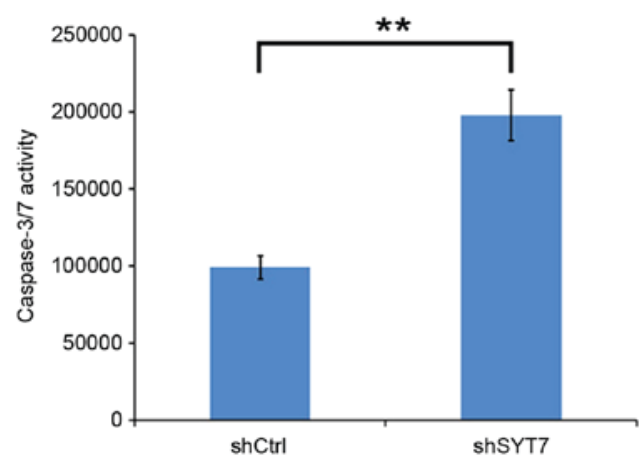

Figure 6. Effect of SYT7 on caspase-3/7 activity. U87MG cells were infected with the shSYT7 lentivirus or shCtrl lentivirus. A total of 3 days following infection, the activity of caspase-3/7 was significantly increased in the shSYT7 group compared with the shCtrl group. ${ }^{* *} \mathrm{P}<0.01$. sh, short hairpin; SYT7, synaptotagmin-7; Ctrl, control.

caspase-3/7 assay and an apoptosis assay using flow cytometry were performed. It was observed that the activity of caspase-3/7 was significantly increased in the shSYT7 group compared with the shCtrl group (Fig. 6). The results of the flow cytometry analysis indicated that U87MG cellular apoptosis was significantly increased in the shSYT7 group compared with the shCtrl group (Fig. 7). Therefore, the results of the present study demonstrated that the downregulation of SYT7 suppressed cell proliferation by promoting cellular apoptosis. 

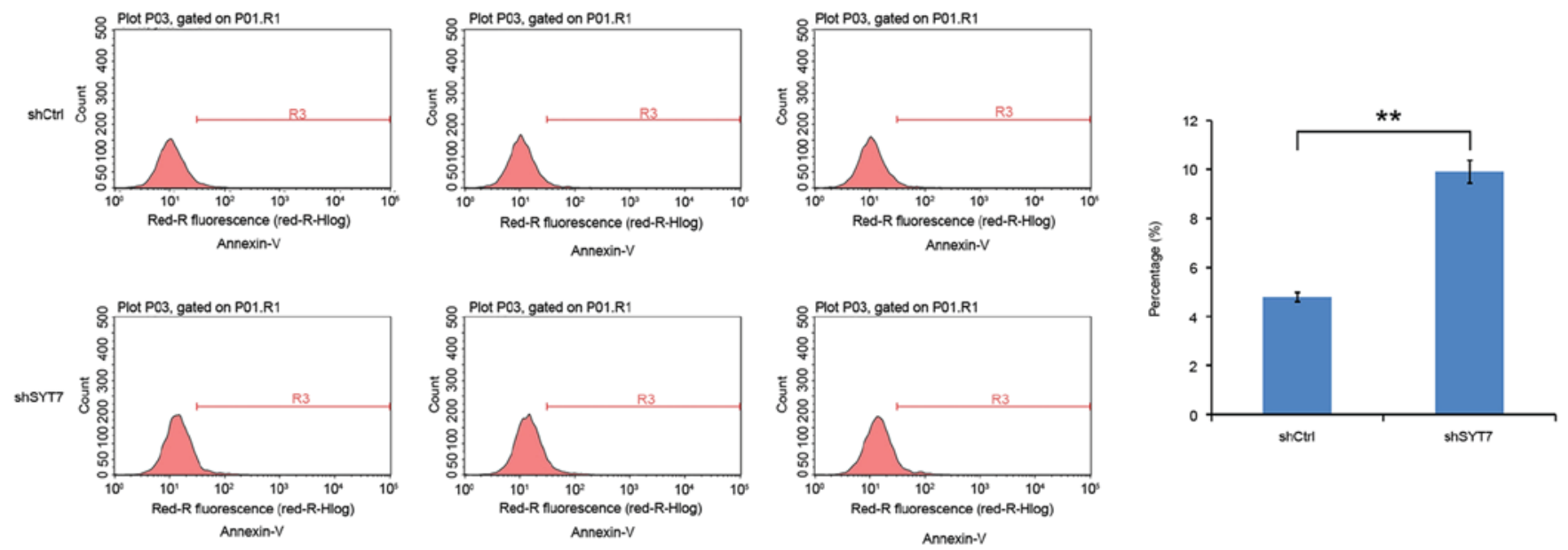

Figure 7. Effect of SYT7 on apoptosis, as demonstrated by flow cytometry. U87MG cells were infected with the shSYT7 lentivirus or shCtrl lentivirus. U87MG cellular apoptosis was significantly increased in the shSYT7 group compared with the shCtrl group. ${ }^{* *} \mathrm{P}<0.01$. sh, short hairpin; SYT7, synaptotagmin-7; Ctrl, control.

\section{Discussion}

In the present study, it was demonstrated that the downregulation of SYT7 inhibited glioblastoma growth by promoting cellular apoptosis. The expression levels of SYT7 were examined in three glioma cell lines (A172, U251 and U87), and it was observed that SYT7 was expressed in all the cell lines; however, only the mRNA levels were assayed, and the expression of SYT7 in glioblastoma multiforme or high-grade glioma tumor samples requires further study. Western blotting or an immunohistochemistry assay may be used to determine the SYT7 expression. In the present study, the target knockdown efficiency in 293T cells was determined using western blotting. The SYT7 protein level was downregulated by shSYT7, and shSYT7 worked efficiently in U87MG cells. An MTT assay and Celigo Image Cytometer Analysis were performed for the examination of cell proliferation, and it was observed that U87MG cell proliferation was significantly inhibited by shSYT7. In addition, a caspase-3/7 assay and an apoptosis assay by flow cytometry were performed to examine the mechanism of cell growth inhibition. It was observed that the activity of caspase-3/7 and U87MG cellular apoptosis was significantly increased in shSYT7 group. However, it is important to note that these conclusions are taken from a single cell line and only the potential role of SYT7 in cellular apoptosis was assessed; further in vitro assays are required to determine the biological role of SYT7 in mitochondrial function, for example.

Glioma is the most common type of human cerebral tumor, with a highly malignant character and poor prognosis (19). The primary treatment for glioblastoma is surgery combined with postoperative radiotherapy, chemotherapy and immunotherapy. As progress in the biological research and treatment of glioblastoma has been made, increasing numbers of gene therapies have been trialed in glioblastoma, including anti-angiogenesis (20), hypoxic tumor-targeting therapy (21) and RNA interference treatment (22), which have achieved promising results.

SYT is a protein sensor of calcium signaling (5-8); it is able to interact with SNARE to regulate the fusion of vesicle membranes and the plasma membrane, serving important roles in cellular secretion (12). The expression of SYT7 is associated with prostate cancer and serves an important role in prostate cancer development (15). However, the SYT family has numerous members which are widely distributed and exhibit complex functions (23). SYT 7 is considered to be the main $\mathrm{Ca}^{2+}$ sensor of synaptic vesicles $(13,24)$. Lysosomes are an essential component of plasma membrane repair. The exocytosis of lysosomes is induced by $\mathrm{Ca}^{2+}$ and depends on the function of SYT7 (14). The possible mechanism of action of SYT7 in glioma involves the downregulation of SYT7 which leads to the inhibition of lysosome-dependent plasma membrane repair, thereby inducing apoptosis and inhibiting cell proliferation. However, the molecular mechanism requires further clarification. The intracellular localization of other subtypes of SYT remains to be elucidated, and their physiological function and interactions with other proteins remain unclear. Further research is required into the physiological function and molecular mechanisms of SYT proteins.

In conclusion, the present study demonstrated that the downregulation of SYT7 inhibited glioblastoma growth by promoting cellular apoptosis. SYT7 may be considered to be a novel therapeutic target for glioblastoma. However, the mechanism underlying the effects of SYT7 in glioblastoma remains to be elucidated, and more in-depth research is required.

\section{Acknowledgements}

The present study was supported by the National Natural Science Foundation of China (grant no. 81660420), the Construction Plan of the Superior Science and Technology Innovation Team of Jiangxi Province (grant no. 20152BCB24009), the Foreign Science and Technology Cooperation Plan of Jiangxi Province (grant no. 20151BDH80009), the Traditional Chinese Medicine Scientific Research Project of Jiangxi Province Health and Family Planning Commission (grant no. 2014A103) and the Guiding Science and Technology Project of Nanchang [grant no. 2014 (196)]. 


\section{References}

1. Krex D, Klink B, Hartmann C, von Deimling A, Pietsch T, Simon M, Sabel M, Steinbach JP, Heese O, Reifenberger G, et al: Long-term survival with glioblastoma multiforme. Brain 130: 2596-2606, 2007.

2. Xiao B, Zhou X, Ye M, Lv S, Wu M, Liao C, Han L, Kang C and Zhu X: MicroRNA-566 modulates vascular endothelial growth factor by targeting Von Hippel-Landau in human glioblastoma in vitro and in vivo. Mol Med Rep 13: 379-385, 2016.

3. Wu M, Fan Y, Lv S, Xiao B, Ye M and Zhu X: Vincristine and temozolomide combined chemotherapy for the treatment of glioma: A comparison of solid lipid nanoparticles and nanostructured lipid carriers for dual drugs delivery. Drug Deliv 23 2720-2725, 2016.

4. Alves TR, Lima FR, Kahn SA, Lobo D, Dubois LG, Soletti R, Borges H and Neto VM: Glioblastoma cells: A heterogeneous and fatal tumor interacting with the parenchyma. Life Sci 89: 532-539, 2011.

5. Rickman C, Craxton M, Osborne S and Davletov B: Comparative analysis of tandem $\mathrm{C} 2$ domains from the mammalian synaptotagmin family. Biochem J 378: 681-686, 2004.

6. Sugita S, Shin OH,Han W,Lao Y and Südhof TC: Synaptotagmins form a hierarchy of exocytotic $\mathrm{Ca}(2+)$ sensors with distinct $\mathrm{Ca}(2+)$ affinities. EMBO J 21: 270-280, 2002.

7. Rizo J, Chen X and Arac D: Unraveling the mechanisms of synaptotagmin and SNARE function in neurotransmitter release. Trends Cell Biol 16: 339-350, 2006.

8. Gustavsson N and Han W: Calcium-sensing beyond neurotransmitters: Functions of synaptotagmins in neuroendocrine and endocrine secretion. Biosci Rep 29: 245-259, 2009.

9. Vasquez JK, Chantranuvatana K, Giardina DT, Coffman MD and Knight JD: Lateral diffusion of proteins on supported lipid bilayers: Additive friction of synaptotagmin $7 \mathrm{C} 2 \mathrm{~A}-\mathrm{C} 2 \mathrm{~B}$ tandem domains. Biochemistry 53: 7904-7913, 2014.

10. Osborne SL, Herreros J, Bastiaens PI and Schiavo G: Calcium-dependent oligomerization of synaptotagmins I and II Synaptotagmins I and II are localized on the same synaptic vesicle and heterodimerize in the presence of calcium. J Biol Chem 274: 59-66, 1999.

11. Andrews NW and Chakrabarti S: There's more to life than neurotransmission: The regulation of exocytosis by synaptotagmin VII. Trends Cell Biol 15: 626-631, 2005.

12. Yoshihara M and Montana ES. The synaptotagmins: Calcium sensors for vesicular trafficking. Neuroscientist 10: 566-574, 2004.
13. Dlugosz A, Muschiol S, Zakikhany K, Assadi G, D'Amato M and Lindberg G: Human enteroendocrine cell responses to infection with Chlamydia trachomatis: A microarray study. Gut Pathog 6: 24, 2014.

14. Divangahi M, Chen M, Gan H, Desjardins D, Hickman TT, Lee DM, Fortune S, Behar SM and Remold HG: Mycobacterium tuberculosis evades macrophage defenses by inhibiting plasma membrane repair. Nat Immunol 10: 899-906, 2009.

15. Walker MG, Volkmuth W, Sprinzak E, Hodgson D and Klingler T: Prediction of gene function by genome-scale expression analysis: Prostate cancer-associated genes. Genome Res 9: 1198-1203, 1999.

16. Allen M, Bjerke M, Edlund H, Nelander S and Westermark B: Origin of the U87MG glioma cell line: Good news and bad news. Sci Transl Med 8: 354re3, 2016.

17. Livak KJ and Schmittgen TD: Analysis of relative gene expression data using real-time quantitative PCR and the 2(-Delta Delta C(T)) method. Methods 25: 402-408, 2001.

18. Liu H, Liang S, Yang X, Ji Z, Zhao W, Ye X and Rui J: RNAi-mediated RPL34 knockdown suppresses the growth of human gastric cancer cells. Oncol Rep 34: 2267-2272, 2015.

19. Xiong J, Bing Z, Su Y, Deng D and Peng X: An integrated mRNA and microRNA expression signature for glioblastoma multiforme prognosis. PLoS One 9: e98419, 2014.

20. Tanaka T, Manome Y, Wen P, Kufe DW and Fine HA: Viral vector-mediated transduction of a modified platelet factor 4 cDNA inhibits angiogenesis and tumor growth. Nat Med 3: 437-442, 1997.

21. Dupont F, Avalosse B, Karim A, Mine N, Bosseler M, Maron A, Van den Broeke AV, Ghanem GE, Burny A and Zeicher M: Tumor-selective gene transduction and cell killing with an oncotropic autonomous parvovirus-based vector. Gene Ther 7: 790-796, 2000

22. Kang CS, Pu PY, Li YH, Zhang ZY, Qiu MZ, Huang Q and Wang GX: An in vitro study on the suppressive effect of glioma cell growth induced by plasmid-based small interference RNA (siRNA) targeting human epidermal growth factor receptor. J Neurooncol 74: 267-273, 2005.

23. Hui E, Bai J, Wang P, Sugimori M, Llinas RR and Chapman ER: Three distinct kinetic groupings of the synaptotagmin family: Candidate sensors for rapid and delayed exocytosis. Proc Natl Acad Sci USA 102: 5210-5214, 2005.

24. Maximov A and Südhof TC: Autonomous function of synaptotagmin 1 in triggering synchronous release independent of asynchronous release. Neuron 48: 547-554, 2005. 